\title{
Anomalous cosmic ray oxygen gradients throughout the heliosphere
}

A. C. Cummings ${ }^{1}$, R. A. Mewaldt ${ }^{1}$, J. B. Blake ${ }^{2}$, J. R. Cummings ${ }^{1}$, M. Fränz ${ }^{3}$, D. Hovestadt ${ }^{4}$, B. Klecker ${ }^{4}$, G. M. Mason ${ }^{5}$, J. E. Mazur ${ }^{5}$, E. C. Stone ${ }^{1}$, T. T. von Rosenvinge ${ }^{6}$, and W. R. Webber ${ }^{7}$

\begin{abstract}
We have used data from the SAMPEX, Ulysses, Voyager 1, Voyager 2, and Pioneer 10 spacecraft to determine the radial and latitudinal gradients of anomalous cosmic ray oxygen at $10 \mathrm{MeV} /$ nuc during the last half of 1993. These five spacecraft cover radial distances from $1 \mathrm{AU}$ (SAMPEX) to $58 \mathrm{AU}$ (P10) and latitudes to $41^{\circ} \mathrm{S}$ (Ulysses) and $32^{\circ} \mathrm{N}$ (V1). We find that the radial gradient is a decreasing function of radial distance, $\sim r^{-n}$, with $\mathrm{n}=1.7 \pm 0.7$. The large-scale radial gradient between the inner and outer heliosphere is much smaller than it was during the last solar minimum period in $\sim 1987$. The latitudinal gradient is small and positive, $1.3 \pm 0.4 \% / \mathrm{deg}$, as opposed to the large and negative latitudinal gradients found during 1987 , but similar to the small positive latitudinal gradient measured during 1976 for anomalous cosmic ray helium. These observations confirm that effects of curvature and gradient drift in the large scale magnetic field of the Sun are important for establishing the three-dimensional intensity distributions of these particles in the heliosphere during periods of solar minimum conditions.
\end{abstract}

\section{Introduction}

Just over twenty years ago, observations of the energy spectra of quiet-time cosmic rays with $\sim 5$ to 50 $\mathrm{MeV} /$ nuc led to the discovery of a new component of cosmic rays (see, e.g., review in Jokipii [1990]). The anomalous cosmic ray (ACR) component, so named because of its unusual composition and spectral shape, is characterized by anomalous enhancements in the energy spectra of the elements $\mathrm{He}, \mathrm{C}, \mathrm{N}, \mathrm{O}, \mathrm{Ne}$, and Ar. Evidence has also been presented for anomalous $\mathrm{H}$ [Christıan et al., 1988], but this has been more controversial until recent confirmation by Voyager in

\footnotetext{
${ }^{1}$ California Institute of Technology, Pasadena

${ }^{2}$ Aerospace Corp., El Segundo

${ }^{3}$ MPI für Aeronomie, Katlenburg Lindau, FRG

${ }^{4}$ MPI für Extraterrestrische Physik, Garching, FRG

${ }^{5}$ University of Maryland, College Park

${ }^{6}$ Goddard Space Flight Center, Greenbelt

${ }^{7}$ New Mexico State University, Las Cruces
}

Copyright 1995 by the American Geophysical Union.

Paper number 94GL03343

0094-8534/95/94GL-03343\$03.00
1994 (e.g., Stone et al., [1994]). ACRs are thought to be interstellar neutrals [Fisk et al., 1974] which flow into the heliosphere, become ionized by either solar UV or solar wind protons in the interplanetary medium, and are then accelerated in the outer heliosphere, probably at the solar wind termination shock [Pesses et al., 1981].

According to the theories of particle acceleration and transport which include curvature and gradient drifts in the large-scale heliospheric magnetic field, the spatial distribution of ACRs near solar minimum should depend on the orientation of the Sun's field. When the Sun's magnetic field is directed inward at its North pole, the so-called $\mathrm{A}<0$ period, which last occurred during the 1987 solar minimum and recurs each 22 years, positive particles gain access to the inner heliosphere via rapid inward drift along the neutral sheet. However, during $\mathrm{A}>0$ periods, appropriate to the current study, the particles flow down onto the heliographic equatorial plane from the polar regions [Jokipir et al., 1977]. This leads to two predicted effects: a latitudinal gradient which reverses sign between the two periods and a radial gradient which is smaller in the $A>0$ phase than in the $\mathrm{A}<0$ phase of the solar cycle.

A key test of theories which include "drifts" is a determination of the sign of the latitudinal gradient in the two phases of the solar cycle. During $\mathrm{A}<0$ periods, the latitudinal gradient should be negative, as was observed in 1987 [Cummings et al., 1990], and during $\mathrm{A}>0$ periods, the latitudinal gradient should be positive. A small positive latitudinal gradient of ACR helium was observed in 1976 [McKibben, 1989], in agreement with the theory. In this paper we will determine the radial and latitudinal gradients of ACR oxygen during the current $\mathrm{A}>0$ period using five widely spaced spacecraft: SAMPEX in near-Earth orbit, Ulysses at $41^{\circ} \mathrm{S}$ heliolatitude, Voyager 1 (V1) at $32^{\circ} \mathrm{N}$ and $54 \mathrm{AU}$, Voyager 2 (V2) at $10^{\circ} \mathrm{S}$ and $41 \mathrm{AU}$, and Pioneer 10 (P10) at $3^{\circ} \mathrm{N}$ and $58 \mathrm{AU}$. We assume symmetry of the particle distributions about the heliographic equator, in accordance with the drift theory.

\section{Observations}

This analysis includes data collected during the period $1993 / 183$ - 365, a period near solar minimum when the intensity of ACRs at $1 \mathrm{AU}$ was already near the level recorded during the 1972 - 1978 solar minimum [Mewaldt et al., 1993]. Figure 1 shows the observed energy spectrum of carbon and oxygen at $1 \mathrm{AU}$ as derived from three instruments on SAMPEX (see Baker 


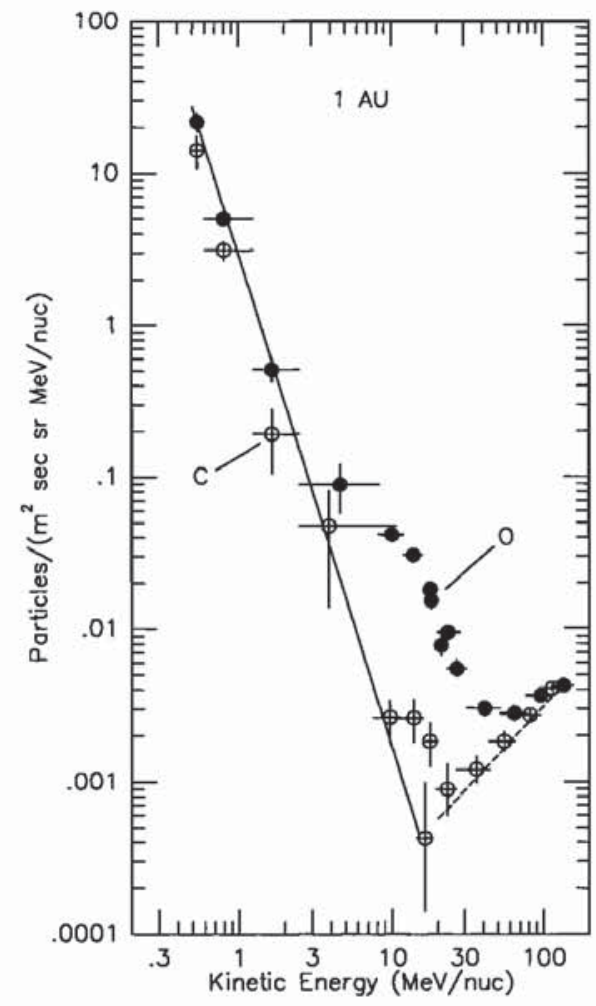

Figure 1. Observed energy spectra of carbon (open circles) and oxygen (filled circles) at $1 \mathrm{AU}$ from three instruments on the SAMPEX spacecraft for the period $1993 / 183-365$. The data were acquired when the spacecraft was over the Earth's magnetic poles (magnetic latitudes $\Lambda>70^{\circ}$ ) during "quiet" times. The solid line is an estimate of low-energy non-ACR O flux as described in the text. The dashed line is an estimate of GCR $O$ as described in the text.

et al. [1993] and companion papers). Note that at high energies the fluxes of carbon and oxygen approach each other, consistent with the composition of galactic cosmic rays (GCRs). At the lowest energies in Figure 1 , a non-ACR component due to perhaps solar or interplanetary particles dominates. The middle energy range of the oxygen spectrum is dominated by ACR oxygen. Estimates of the energy spectrum of GCR oxygen and of low-energy solar or interplanetary oxygen are shown in Figure 1. The GCR spectrum was estimated by fitting a power-law to the four highest energy intervals of the carbon spectrum and adjusting by the factor $1 / 0.89$ to account for the GCR C/O ratio. The low-energy spectrum is a power-law fit to the three lowest-energy oxygen points. The energy spectrum of ACR oxygen is obtained by subtracting these two "background" spectra from the observed spectrum.

It is possible that there is an additional solar or interplanetary component in Figure 1 in the 10 to 15 $\mathrm{MeV} /$ nuc energy range as suggested by the "turn-up" in the carbon energy spectrum (see Mewaldt et al. [1993] for more discussion). The $\mathrm{C} / \mathrm{O}$ ratio at these energies is small, $\sim 0.1$, but much larger than the ACR C/O ratio, $\sim 0.01$, observed in the outer heliosphere on Voyager [Cummings and Stone, 1990]. We have not corrected for a possible background of $\mathrm{O}$ particles associated with this probable solar/interplanetary component as the correction would be insignificant at $10 \mathrm{MeV} / \mathrm{nuc}$, the energy at which we will compare fluxes.

In Figure 2 we show energy spectra of ACR oxygen at V1, V2, P10, Ulysses, and SAMPEX for the period 1993/183-365. (The Ulysses EPAC instrument is described in Keppler et al. [1992].) Where appropriate, subtractions for GCR and low-energy components described above have been made before plotting. We have chosen $10 \mathrm{MeV} /$ nuc as the energy for comparing fluxes at the 5 spacecraft because the overlap in spectral coverage, though not perfect, is approximately optimum there. For all energy spectra, except for the one from Ulysses, the flux at $10 \mathrm{MeV} /$ nuc was interpolated by using a power law.

The oxygen energy spectrum from Ulysses extends only up to $6 \mathrm{MeV} /$ nuc. For an estimate of the upper limit of the flux at $10 \mathrm{MeV} /$ nuc we extrapolated by using a power-law fit to the three Ulysses points. This should be a conservative upper limit since the spherically-symmetric model spectra of Reinecke et al. [1993] have peak energies near 5-6 MeV/nuc. To address the lower limit we used the calculated ratio of 0.72 for ACR $\mathrm{O}$ flux at $10 \mathrm{MeV} /$ nuc to that at $6 \mathrm{MeV} / \mathrm{nuc}$ for the Rernecke et al. model spectrum at $2 \mathrm{AU}$ for

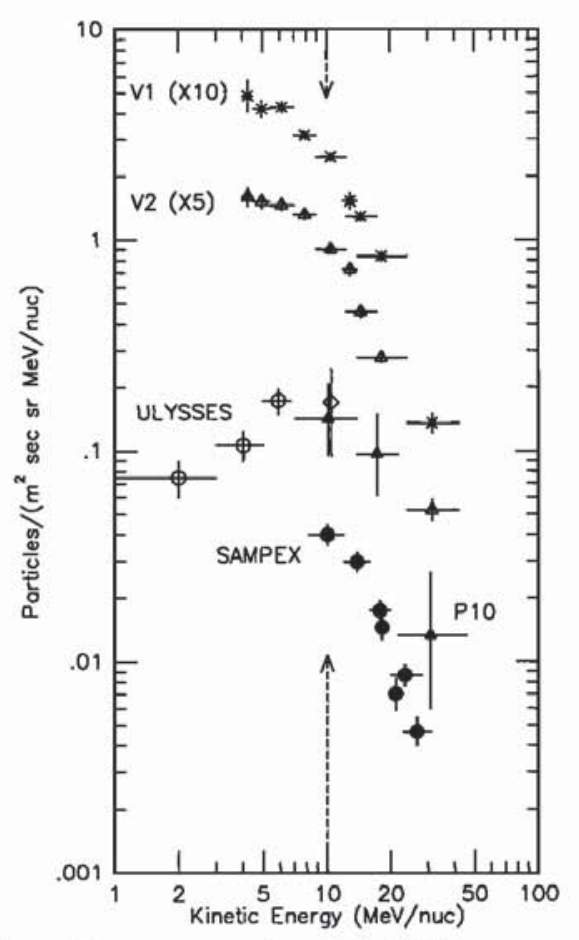

Figure 2. Energy spectra of ACR oxygen at the position of 5 spacecraft for the period 1993/183 - 365. The heliographic radial and latitudinal positions of the spacecraft are given in Table 1 . The two vertical dashed lines with arrows drawn at $10 \mathrm{MeV} /$ nuc indicate the energy of comparison between the 5 spacecraft. For Ulysses the data were acquired during the "low $\mathrm{He}$ threshold" periods as described in Blake et al. [submitted to Cospar Colloq. Ser., 1994]. An estimate of the flux of ACR O from Ulysses is shown as the open diamond symbol and its derivation is described in the text. 
the 1978 period (bottom dashed curve in their Figure $3 \mathrm{a})$ and applied it to the observed Ulysses flux at 6 $\mathrm{MeV} /$ nuc. To establish the lower limit we assumed a systematic uncertainty of $20 \%$ on the ratio from the model. Since the fitting procedure employed requires equal uncertainties on the points, we have taken the average of the upper and lower limits as our estimate of the flux of ACR $\mathrm{O}$ at $10 \mathrm{MeV} / \mathrm{nuc}$ at Ulysses. This point is shown as the open diamond symbol in Figure 2. Although this procedure results in a large uncertainty compared to the statistical uncertainty associated with the observed Ulysses flux at $6 \mathrm{MeV} /$ nuc, we feel it reflects an appropriate uncertainty in characterizing the shape of the expected energy spectrum at $41^{\circ} \mathrm{S}$ heliolatitude.

Table 1 shows the average heliographic radii and latitudes of all 5 spacecraft during this period and the fluxes of ACR oxygen at $10 \mathrm{MeV} /$ nuc at each position. The fluxes are plotted versus radial position in Figure 3. The slope of the dashed line in Figure 3 is derived from 7.1 - $17.1 \mathrm{MeV} /$ nuc measurements at low heliographic latitudes in the inner heliosphere in 1978 [Webber et al., 1985]. The intensity of the dashed curve has been adjusted to match the current observations at $1 \mathrm{AU}$.

The solid line in Figure 3 is the intensity of ACR $\mathrm{O}$ derived from the fluxes at the three spacecraft in the outer heliosphere, assuming constant radial and latitudinal gradients (see Cummings et al. [1987] for details of the method). The curve has been normalized to the flux at P10. The deduced radial and latitudinal gradients from this method are $-1.0 \pm 1.6 \% / \mathrm{AU}$ and $1.9 \pm 0.9 \% / \mathrm{deg}$. We note that the uncertainty on the radial gradient does not exclude a small positive value, which would be expected if the source region is beyond $\mathrm{P} 10$. If the radial gradient in the outer heliosphere is zero, then the latitudinal gradient is $1.3 \pm 0.4 \% / \mathrm{deg}$ (from V1 and V2 measurements).

The solid and dashed curves in Figure 3 suggest that the radial gradient is larger in the inner heliosphere than in the outer heliosphere, similar to the situation for the 1987 solar minimum [Cummings et al., 1990]. Figure 4a and $b$ shows least-squares fits (based on the LevenbergMarquardt method [Press et al., 1992]) to the ACR oxygen fluxes assuming a constant latitudinal gradient

Table 1. Gradient Parameters for 1993/183-365

\begin{tabular}{|c|c|c|c|}
\hline \multirow[b]{2}{*}{$\mathrm{S} / \mathrm{C}$} & \multicolumn{2}{|c|}{ Heliographic } & \multirow{2}{*}{$\begin{array}{c}\text { ACR O Flux } \\
@ 10 \mathrm{MeV} / \text { nuc } \\
\left(\mathrm{m}^{2} \mathrm{~s} \mathrm{sr} \mathrm{MeV/nuc}\right)^{-1}\end{array}$} \\
\hline & $\begin{array}{l}\text { Rad. } \\
\text { (AU) }\end{array}$ & $\begin{array}{c}\text { Lat. }^{a} \\
\text { deg }\end{array}$ & \\
\hline SAMPEX & 1.0 & $4.7^{b}$ & $(3.98 \pm 0.43) \times 10^{-2}$ \\
\hline Ulysses & 4.2 & -41.1 & $(1.70 \pm 0.76) \times 10^{-1}$ \\
\hline V2 & 41.1 & -10.2 & $(1.91 \pm 0.11) \times 10^{-1}$ \\
\hline V1 & 53.6 & 32.3 & $(2.57 \pm 0.14) \times 10^{-1}$ \\
\hline P10 & 58.0 & 3.2 & $(1.42 \pm 0.65) \times 10^{-1}$ \\
\hline
\end{tabular}

${ }^{a}$ Negative latitudes are South.

${ }^{b}$ Average of absolute value of latitude over time period.

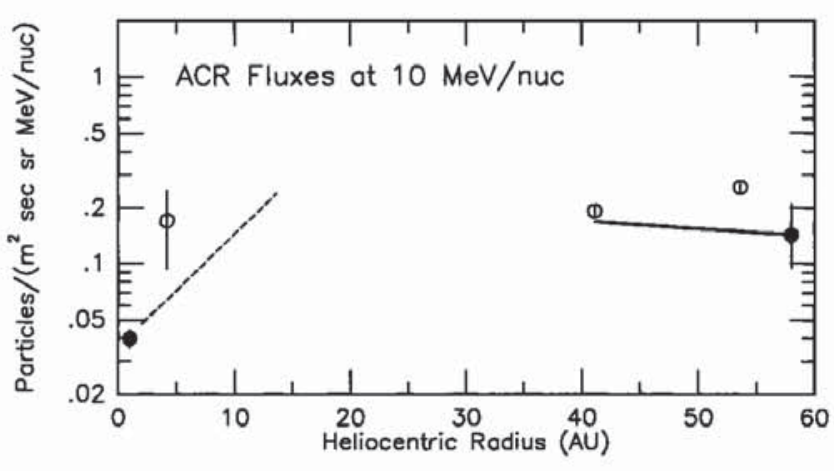

Figure 3. Flux of $\mathrm{ACR} O$ at $10 \mathrm{MeV} /$ nuc from Table 1 versus heliographic radius. The solid and dashed lines are rough estimates of the radial gradient as described in the text. The open circles denote spacecraft (Ulysses, V1, and V2) that are significantly off the heliographic equator.

and a differential radial gradient, $g_{r}=(1 / f)(\partial f / \partial r)$, which is proportional to $r^{-n}$. This is the same form used by Cummings et al. [1990] to describe the 1987 data, which is reproduced in Figure $4 \mathrm{c}$ and $\mathrm{d}$. Panels a and $c$ of Figure 4 show the radial dependence of the flux after correcting for the latitudinal gradient,

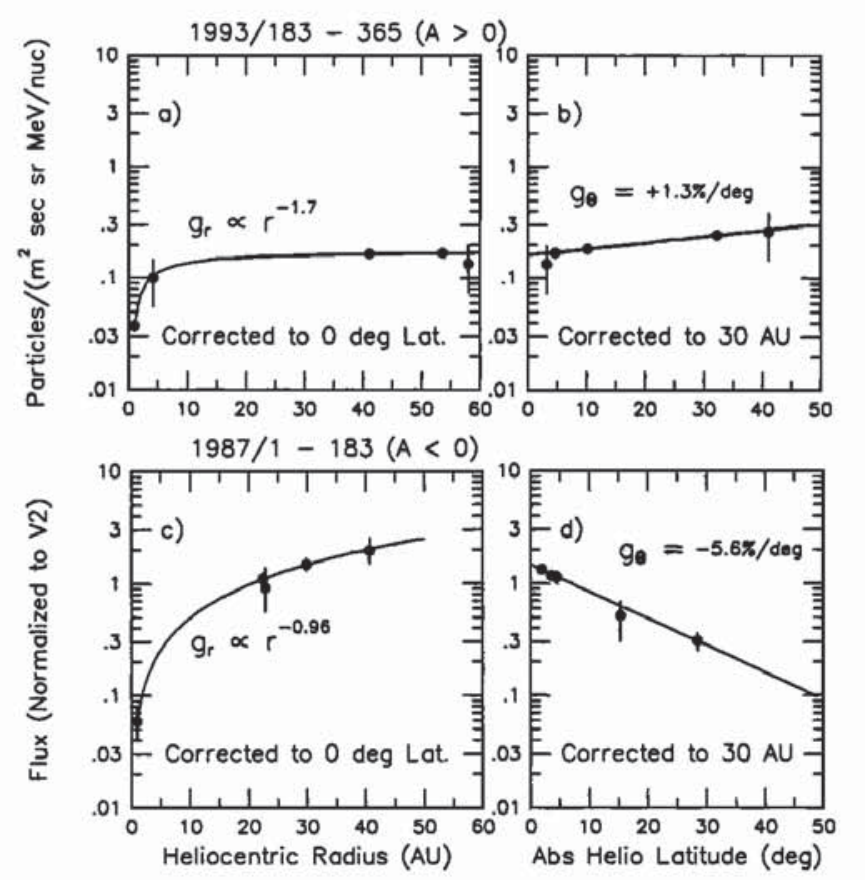

Figure 4. (a) Flux of ACR $\mathrm{O}$ at $10 \mathrm{MeV} /$ nuc versus heliographic radial distance for the time period $1993 / 183$ - 365, corresponding to $A>0$. The fluxes have been corrected to $0^{\circ}$ using a latitudinal gradient of $1.3 \% / \mathrm{deg}$. The solid line is a least-squares fit to the data as described in the text. (b) Fluxes as in (a), except plotted versus heliographic latitude and corrected to 30 AU using parameters from the least-squares fit to the data shown as the solid line. (c) Same as (a) above except energy interval is $7-25 \mathrm{MeV} /$ nuc and time period is $1987 / 1-183$, corresponding to $A<0$. The latitudinal gradient used to correct the fluxes to $0^{\circ}$ is $5.6 \% /$ deg. (d) Same as (b) above except for $1987 / 1-183$ time period. Panels (c) and (d) are reproduced from Cummings et al. [1990]. 
and panels $\mathrm{b}$ and $\mathrm{d}$ show the latitudinal dependence of the flux corrected to $30 \mathrm{AU}$. The best-fit value of $\mathrm{n}$ for 1993 is $1.7 \pm 0.7$ and the latitudinal gradient is $1.3 \pm 0.4 \% / \mathrm{deg}$. This value of the latitudinal gradient is consistent with the value derived above from the three spacecraft in the outer heliosphere, supporting the assumption that the latitudinal gradient is independent of radius. The average large-scale radial gradient in 1993 between 1 and $58 \mathrm{AU}$ is quite small, $2.2 \pm 0.8 \% / \mathrm{AU}$, compared to $8.8 \pm 0.6 \% / \mathrm{AU}$ observed in 1987 between 1 and $41 \mathrm{AU}$.

\section{Discussion}

The small positive latitudinal gradient observed in this study contrasts sharply with the large negative latitudinal gradient observed during 1987 (compare Figure $4 \mathrm{~b}$ and d). In 1976, however, when the Sun's magnetic polarity was the same as now, a small positive latitudinal gradient of ACR helium, $\sim 2 \% / \mathrm{deg}$, was observed based on $\mathrm{P} 11$ observations at $16^{\circ} \mathrm{N}$ [McKibben, 1989]. Thus we now have evidence that the latitudinal gradient reverses sign in three consecutive solar magnetic field epochs, giving strong support to theories of particle propagation that include drifts in the largescale magnetic field [Jokipii et al., 1990].

In addition, the magnitude of the average radial gradient from the inner to the outer heliosphere is a factor of $\sim 4$ smaller in 1993 than in 1987. This is also in agreement with predictions based on the drift theory because in the present $\mathrm{A}>0$ period the particles are expected to be drifting down from the poles onto the heliographic equatorial plane.

In 1994 Ulysses reached its maximum southerly excursion in latitude (about $80^{\circ} \mathrm{S}$ ). It will reach a similar northerly latitude one year later. We plan to continue these multi-spacecraft observations as Ulysses rapidly traverses $\sim 160^{\circ}$ in heliographic latitude.

Acknowledgments. This work was supported by NASA under contracts NAS7-918, NAS5-30704, JPL contract 958748, and Agreement 26979B. This work was also supported by the MPG and BMFT under grant numbers $01 \mathrm{ON} 1990,50 \mathrm{ON} 87037,50 \mathrm{OC} 90021$, and 50ON91050.

\section{References}

Baker, D. N., et al., An overview of the solar, anomalous, and magnetospheric particle explorer (SAMPEX) mission, IEEE Trans. Geosci. Remote Sensing, 31, 531, 1993.

Christian, E. R., A. C. Cummings, and E. C. Stone, Evidence for anomalous cosmic ray hydrogen, Astrophys. J. Lett., 334, L77, 1988.

Cummings, A. C., E. C. Stone, and W. R. Webber, Latitudinal and radial gradients of anomalous and galactic cosmic rays in the outer heliosphere, Geophys. Res. Lett., $14,174,1987$.

Cummings, A. C., and E. C. Stone, Elemental composition of the very local interstellar medium as deduced from observations of anomalous cosmic rays, Proc. Int. Conf. Cosmic Ray 21st, 6, 202, 1990.

Cummings, A. C., et al., Radial and latitudinal gradients of anomalous cosmic-ray oxygen and helium from 1 to $\sim 41$ AU, Proc. Int. Conf. Cosmic Ray 21st, 6, 206, 1990.

Fisk, L., B. Kozlovsky, and R. Ramaty, An interpretation of the observed oxygen and nitrogen enhancements in lowenergy cosmic rays, Astrophys. J. Lett., 190, L35, 1974.

Jokipii, J. R., E. H. Levy, and W. R. Hubbard, Effects of particle drift on cosmic-ray transport. I. general properties, applications to solar modulation, Astrophys. J., 213, 861, 1977.

Jokipii, J. R., The anomalous component of cosmic rays, in Physics of the Outer Heliosphere, Cospar Colloq. Ser., edited by S. Grzedielski and D. E. Page, Pergamon, New York, 169, 1990.

Keppler, E., et al., The Ulysses energetic particle composition experiment EPAC, Astron. Astrophys. Suppl. Ser., 92, 317, 1992.

McKibben, R. B., Reanalysis and confirmation of positive latitude gradients for anomalous helium and galactic cosmic rays measured in 1975-1976 with Pioneer 11, J. Geophys. Res., 94, 17021, 1989.

Mewaldt, R. A., et al., The return of the anomalous cosmic rays to $1 \mathrm{AU}$ in 1992, Geophys. Res. Lett., 20, 2263, 1993.

Pesses, M. E., J. R. Jokipii, and D. Eichler, Cosmic ray drift, shock wave acceleration, and the anomalous component of cosmic rays, Astrophys. J. Lett., 246, L85, 1981.

Press, W. H., et al., Numerical recipes in $\mathrm{C}$ the art of scientific computing, Cambridge University Press, 2nd Edition, 683, 1992.

Reinecke, J. P. L., H. Moraal, and F. B. McDonald, The cosmic radiation in the heliosphere at successive solar minima: steady state no-drift solutions of the transport equation, J. Geophys. Res., 98, 9417, 1993.

Stone, E. C., A. C. Cummings, and W. R. Webber, Energy spectra of anomalous cosmic rays in the outer heliosphere (abstract), Eos Trans. AGU, 75 (Suppl.), 530, 1994.

Webber, W. R., A. C. Cummings, and E. C. Stone, Radial and latitudinal gradients of anomalous oxygen during 1977-1985, Proc. Int. Conf. Cosmic Ray 19th, 5, 172, 1985.

A. C. Cummings, R. A. Mewaldt, J. R. Cummings, and E. C. Stone, California Institute of Technology, Mail Code 220-47, Pasadena, CA 91125.

J. B. Blake, Aerospace Corp., El Segundo, CA 91125.

M. Fränz, MPI für Aeronomie, Katlenburg Lindau, FRG.

B. Klecker and D. Hovestadt, MPI für Extraterrestrische Physik, Garching, FRG.

W. R. Webber, Astronomy Dept., New Mexico State University, Las Cruces, NM 88003.

G. M. Mason and J. E. Mazur, University of Maryland, College Park, MD 20742.

T. T. von Rosenvinge, Goddard Space Flight Center, Greenbelt, MD 20771.

(received November 10, 1994; accepted November 28, 1994.) 